\title{
Diseño de una aplicación Web para el seguimiento y control del sobrepeso u obesidad a través del paradigma del IoT
}

\author{
Uriel Ramos-Deonati ${ }^{1}$, Giner Alor-Hernández ${ }^{1}$, Isaac Machorro-Cano ${ }^{1}$, \\ José Luis Sánchez-Cervantes ${ }^{2}$, Lisbeth Rodríguez-Mazahua ${ }^{1}$ \\ ${ }^{1}$ Instituto Tecnológico de Orizaba, División de Estudios de Posgrado e Investigación, \\ Veracruz, México \\ ${ }^{2}$ CONACYT - Instituto Tecnológico de Orizaba, \\ Veracruz, México \\ \{deonatiuriel, imachorro\}@gmail.com, \{galor, lrodriguez\}@itorizaba.edu.mx, \\ jlsanchez@conacyt.mx
}

\begin{abstract}
Resumen. En la actualidad, el sobrepeso y la obesidad son dos problemas de salud globales que afectan la calidad de vida de las personas y, por lo tanto, su vida laboral. El Internet de las cosas (IoT, por sus siglas en inglés) es un nuevo paradigma que está ganando terreno rápidamente en el escenario de la tecnología inalámbrica moderna. La idea básica de este concepto es la presencia en el entorno de diversas cosas $\mathrm{u}$ objetos interconectados, tales como los identificadores de radiofrecuencia (RFID), sensores, actuadores, teléfonos inteligentes, entre otras cosas u objetos. En este trabajo se presenta el diseño de una aplicación Web para contribuir en el seguimiento y control del sobrepeso u obesidad, bajo el paradigma del IoT, incorporando técnicas de inteligencia artificial aplicadas al cuidado de la salud. Adicionalmente, se presenta una arquitectura de componentes y el prototipo inicial, el cual está dividido en cinco capas principales (capa de presentación, capa de integración, capa de servicios en el IoT, capa analítica de datos y capa de datos) que integran el modelo interno del sistema, así como el aspecto de la aplicación. Además, se describen las principales funcionalidades de la aplicación Web, su diseño y un análisis de distintas plataformas de aprendizaje automático para el soporte del sistema de recomendaciones médicas, considerando utilizar el algoritmo de clasificación C4.5 para analizar la información obtenida a través de los dispositivos wearables.
\end{abstract}

Palabras clave: inteligencia artificial, IoT, obesidad, RFID, sobrepeso, wearables.

\section{Design of a Web Application for the Monitoring and Control of Overweight or Obesity through the IoT Paradigm}

Abstract. Currently, overweight and obesity are two global health problems that affect the quality of life of people. The Internet of Things (IoT) is a new 


\begin{abstract}
paradigm that is quickly gaining acceptance on the ecosystem of modern wireless technology. The basic idea of this concept is the presence in the environment of various things or interconnected objects such as radiofrequency identifiers (RFID), sensors, actuators, smart phones, to mention but a few. This work presents the design of a Web-based application for monitoring and controlling critical parameters related to overweight or obesity by using the IoT paradigm and incorporating artificial intelligence techniques. Additionally, a layered architecture design of components and its initial prototype are presented. The architecture is divided into five main layers (presentation layer, integration layer, layer of services in the IoT, analytical data layer and data layer) that integrate the internal model of the system. In addition, the main functionalities of the Web application, the design and an analysis of different machine learning platforms for the support of the medical recommendations system are described, considering the use of the $\mathrm{C} 4.5$ classification algorithm to analyze the information obtained through the Wearables devices.
\end{abstract}

Keywords: artificial intelligence, IoT, obesity, overweight, RFID, wearables.

\title{
1. Introducción
}

La obesidad es un estado patológico caracterizado por la acumulación excesiva de grasa corporal. En 2014, más de 1,900 millones de adultos mayores de 18 años en todo el mundo tenían sobrepeso, más de 600 millones eran obesos y 41 millones de niños menores de 5 años tenían sobrepeso o eran obesos [1]. De hecho, la mayoría de la población mundial vive en países donde el sobrepeso y la obesidad causan la muerte de más personas que la insuficiencia renal. Es por esto que el sobrepeso y la obesidad son problemas que afectan la calidad de vida de las personas, particularmente en el aspecto laboral.

Por otra parte, la atención médica es un área de aplicación importante en el IoT, ya que éste está adaptado para mejorar la calidad del servicio y reducir costos. Actualmente, diversos sensores o dispositivos médicos se utilizan para controlar parámetros médicos, tales como la temperatura corporal, el nivel de glucosa en sangre y la frecuencia cardíaca, entre otros. Los recientes avances en sensores, comunicación inalámbrica y tecnologías de procesamiento son la fuerza impulsora detrás de la aplicación del IoT en los sistemas de atención médica. Últimamente, se están desarrollando sensores corporales portátiles, mejor conocidos como wearables, para monitorear continuamente las actividades o los parámetros de los pacientes en tiempo real. En este contexto, el IoT ofrece a los sistemas de salud una interconexión de los diversos dispositivos heterogéneos para obtener información rápida, completa y precisa de los parámetros de salud de un paciente [2].

Asimismo, los entornos de vida asistida facilitan la vida diaria de las personas con discapacidades y condiciones médicas crónicas. Gracias a la capacidad de los sensores para administrar datos de manera eficiente, es posible proporcionar a los pacientes servicios de vida asistida en tiempo real.

Además, el uso del IoT en el cuidado de la salud contribuye a servicios innovadores, como la recopilación de datos vitales del paciente a través de una red de 
sensores conectados a dispositivos médicos. También, contribuye a la entrega de datos a la nube de un centro médico para su almacenamiento y procesamiento, y garantiza el acceso ubicuo o el intercambio de datos médicos (por ejemplo, registros de salud) [3]. Del mismo modo, los desafíos en el sector salud son una oportunidad para desarrollar e implementar el IoT y así contribuir a la mejora de los servicios.

En este trabajo se propone el diseño de una aplicación Web para contribuir en el seguimiento y control del sobrepeso u obesidad bajo el paradigma del IoT, incorporando técnicas de inteligencia Artificial aplicadas al cuidado de la salud, de tal manera que motive a la participación ciudadana y la responsabilidad del autocuidado. Al maximizar la participación ciudadana en el autocuidado se reduce el número de pacientes que padecen sobrepeso u obesidad y, por lo tanto, minimiza el costo social generado para prevenir y atender a los pacientes que padecen estas enfermedades.

Este trabajo está estructurado de la siguiente manera: la sección 2 presenta los trabajos relacionados con el sobrepeso u obesidad y el IoT; la sección 3 explica la metodología de la investigación utilizada para el desarrollo de la aplicación Web basada en IoT; la sección 4 presenta las funcionalidades del prototipo; finalmente, la sección 5 presenta las conclusiones de la investigación y el trabajo futuro.

\section{Trabajos relacionados}

El uso de dispositivos basados en el IoT está empezando a transformar el estilo de vida de millones de personas y particularmente en las diversas actividades del cuidado de la salud. En este sentido, el IoT está aportando grandes oportunidades para la monitorización, análisis, diagnóstico y recomendaciones de enfermedades como el sobrepeso u obesidad; es por ello que muchos investigadores focalizan sus investigaciones en este tópico. A continuación, se presenta una revisión del estado del arte de los trabajos que usan tecnologías Web e IoT en el cuidado de la salud, particularmente en relación al sobrepeso u obesidad.

Vilallonga et al. [4] presentaron un estudio de seguimiento de pacientes obesos después de la cirugía. Los pacientes consideraron el ahorro de tiempo como muy valioso y concluyeron que visualizar sus gráficos de progreso de manera constante fue muy motivador, esto evita que el cirujano pierda tiempo, esfuerzo y personal en el análisis rutinario de los pacientes, en prácticas como medición de peso, tejido graso, grasa magra e índice de masa corporal, los cuales típicamente se obtienen por medio de la báscula; esta información el paciente la envía desde su hogar y se logra dar un seguimiento de forma cómoda y oportuna obteniendo resultados favorables.

A su vez, Zaragozá et al. [5] describieron una plataforma de monitoreo destinada a establecer una red de sensores para niños obesos bajo tratamiento clínico; además, propuso el sistema ETIOBE, el cual se desarrolló para mejorar el tratamiento, promover los mecanismos de autocontrol y prevenir recaídas en niños obesos con tratamiento clínico. ETIOBE es la plataforma de monitoreo ubicua, ya que permite al clínico obtener información relevante de los pacientes (contextual, fisiológica y psicológica), que permite la adaptación del tratamiento, según la evolución del paciente. 
Vazquez et al. [6] presentaron una arquitectura de salud móvil destinada a prevenir la obesidad infantil mediante la promoción de buenos comportamientos de salud, con un conjunto de aplicaciones mHealth. Las aplicaciones proporcionaron notificaciones y mensajes a los de adultos para mejorar los resultados.

Por otro lado, Kim et al. [7] presentaron el diseño y uso de iN Touch, una aplicación de autogestión móvil para el seguimiento de las observaciones de la vida diaria (ODL) en un programa de entrenamiento en salud para jóvenes de bajos recursos, urbanos y de minorías, con sobrepeso u obesidad.

Además, Alloghani et al. [8] propuso una aplicación de salud móvil con el propósito de aumentar los niveles de conciencia de los padres y los niños sobre los riesgos de la obesidad y ayudarlos a mantener un estilo de vida, de alimentación equilibrado y saludable.

Wibisono y Astawa [9] presentaron un sitio Web y aplicaciones móviles para un programa de pérdida de peso con tecnología de máquina a máquina (M2M), utilizando una escala de peso especial para cargar datos al servidor. El sitio Web o la aplicación de Android contiene básicamente cuatro páginas principales: 1) Home. Muestra el inicio de la aplicación; 2) Historial. Contiene estadísticas del peso del usuario para un rango de tiempo específico: los últimos siete días, el último mes, últimos tres meses o todo el programa; 3) Calorías de Alimentos. Muestra varios tipos de alimentos (indonesio y occidental) y las calorías contenidas, y 4) Información personal. Muestra información del usuario como nombre de usuario, nombre, apellido, correo electrónico, contraseña, sexo, altura, peso inicial, objetivo de peso, nivel de actividad diaria y la imagen del usuario. Esta información se completa previamente con el registro del usuario y se actualiza a través de esta página.

Por otra parte, Aupetit et al. [10] describieron el diseño de un tablero para la visualización de datos biométricos de un campamento de obesidad infantil, en Qatar. Este tablero evaluó el estado de salud de un sujeto frente al grupo, comparándolo con otro. Un experto en salud también proporcionó al sujeto un estado objetivo (por ejemplo, peso) para ser comparado, junto con recomendaciones sobre las actividades a mejorar.

Adicionalmente, Yang et al. [11] desarrollaron un estudio para evaluar la efectividad de la prevención de la obesidad en niños de 10 a 12 años de edad, con un sistema de plataforma móvil llamado HAPPY ME, que es una aplicación de teléfono inteligente junto con un dispositivo portátil, diseñado para mejorar los comportamientos saludables para prevenir la obesidad infantil.

Además, Laing et al. [12] realizaron un estudio de la efectividad de una intervención experimental basada en recomendaciones de dieta por medio de una aplicación inteligente para la pérdida de peso en pacientes mayores de 18 años con sobrepeso u obesidad, concluyendo que las aplicaciones para bajar de peso son útiles para las personas que están listas para controlar las calorías. Sin embargo, para el paciente con sobrepeso promedio, es improbable que la introducción de una aplicación de teléfono inteligente produzca un cambio de peso significativo.

De igual forma, Otto et al. [13] presentaron una arquitectura de hardware y software de un sistema de red de sensores inalámbricos para el monitoreo ambulatorio del estado de salud. El sistema consiste en múltiples nodos sensores que monitorean 
el cuerpo, movimiento y actividad del corazón, utilizando un coordinador de red y un servidor personal; además, usaron una arquitectura del software que se basa en TinyOS, un sistema operativo de código abierto ampliamente utilizado para redes de sensores.

Ahmed et al. [14] presentaron una visión general de los sistemas existentes de monitoreo de la salud, teniendo en cuenta el enfoque del IoT, además de las tendencias recientes y el desarrollo de sistemas de monitoreo de la salud en términos de parámetros y marcos de salud, comunicación inalámbrica y problemas de seguridad, así como identificar limitaciones y ventajas.

Finalmente, Shin [15] definió el nuevo concepto de IoT-learning integrando las tecnologías IoT y el aprendizaje omnipresente, y propuso un programa personalizado de entrenamiento utilizando IoT-learning para el control de peso, el cual proporciona a los usuarios no sólo información personalizada, transmitiendo datos a través de la red y recibiendo la información para analizarla, sino también para proporcionar a los usuarios datos que coinciden con la información personalizada. IoT-learning proporciona a los usuarios (estudiantes) retroalimentación mediante la recopilación de información a través de sensores. El objetivo más importante de este aprendizaje es brindar capacitación personalizada a los usuarios con los datos obtenidos a través de la tecnología de sensores en el IoT. Este documento combina la tecnología de detección en el IoT y los sistemas de aprendizaje.

Como se muestra en la Tabla 1 (integrada por los elementos: a. wearable, b. conectividad, c. interoperabilidad, d. integración, e. parámetros médicos, y f. recomendación médica), los trabajos $[5,6,7,13,14]$ cuentan con la inclusión de parámetros médicos, pero no proporcionan recomendaciones médicas.

Tabla 1. Comparativa de trabajos realizados.

\begin{tabular}{|l|c|c|c|c|c|c|}
\hline \multicolumn{1}{|c|}{ Artículo } & a & b & c & d & e & f \\
\hline Vilallonga et al. [4] & No & Sí & Sí & No & Sí & Sí \\
\hline Zaragozá et al. [5] & Sí & Sí & Sí & Sí & Sí & No \\
\hline Vazquez et al. [6] & No & Sí & Sí & Sí & Sí & No \\
\hline Kim et al. [7] & No & Sí & Sí & No & Sí & No \\
\hline Alloghani et al. [8] & Sí & Sí & Sí & Sí & No & Sí \\
\hline Wibisono y Astawa [9] & No & Sí & Sí & Sí & Sí & Sí \\
\hline Aupetit et al. $[10]$ & Sí & Sí & Sí & No & No & Sí \\
\hline Yang et al. $[11]$ & Sí & Sí & Sí & No & Sí & No \\
\hline Laing et al. $[12]$ & No & No & Sí & No & Sí & Sí \\
\hline Otto et al. [13] & Sí & Sí & No & Sí & Sí & No \\
\hline Ahmed et al. $[14]$ & No & No & No & No & Sí & No \\
\hline Shin [15] & Sí & Sí & No & No & Sí & Sí \\
\hline
\end{tabular}

Por otra parte, en $[8,10]$ sí cuentan con recomendaciones, pero no integran parámetros médicos; sin embrago, [9] cuenta con todos los elementos, pero no usa algún dispositivo vestible para la recolección de información ya que se realiza de 
forma manual, en relación con [14] que no tiene una integración ni realiza recomendaciones médicas. Además, [15] cumple con gran parte de los elementos, sin embargo, no contiene buena interoperabilidad ni integración.

Ante este contexto, al realizar un análisis comparativo se observa que el diseño de la aplicación Web para contribuir en el seguimiento y control del sobrepeso u obesidad bajo el paradigma del IoT propuesto en este trabajo, considera todos los aspectos analizados en la Tabla 1. En la siguiente sección se presenta la metodología de la investigación, la cual está integrada por módulos y capas que se utilizaron para el diseño de la aplicación Web relacioanda con el seguimiento y control del sobrepeso u obesidad bajo el paradigma del IoT.

\section{Metodología de la investigación}

La propuesta del diseño de una aplicación Web para el seguimiento y control del sobrepeso u obesidad aborda un tema prioritario sobre una problemática de salud pública, como lo es el sobrepeso u obesidad, el cual se pretende resolver mediante la conectividad, interoperabilidad e integración de dispositivos en el IoT, e incorporando técnicas de inteligencia artificial, aplicadas al cuidado de la salud. Por ello, la metodología planteada se divide en tres módulos, los cuales se muestran en la Fig. 1.

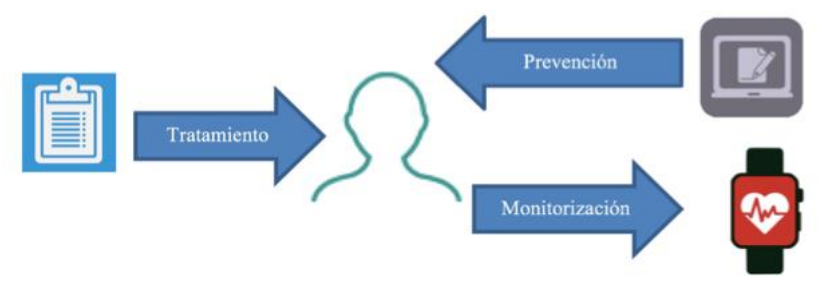

Fig. 1. Metodología de la investigación.

Monitorización. Permitirá medir a través de wearables los signos vitales de las personas con sobrepeso u obesidad para identificar variables críticas (calorías quemadas, sueño, ritmo cardíaco, Índice de Masa Corporal - IMC, número de pisos subidos, calidad del sueño, calorías consumidas, ejercicios, control de peso) y utilizarlas como parámetros para predecir problemas o patologías que afecten la salud de una persona, por medio de técnicas de inteligencia artificial. Además, en este módulo se pretende identificar patrones que mejoren o afecten el sobrepeso $\mathrm{u}$ obesidad en relación a la frecuencia cardíaca, ingesta de alimentos, calorías quemadas, consumo de agua, calidad del sueño, entre otras variables, con el propósito de obtener la información necesaria para la generación de recomendaciones y mensajes de motivación.

Prevención. En este módulo se consideran recomendaciones médicas que conlleven a acciones, procedimientos e intervenciones integrales que mantengan o mejoren la salud de las personas con sobrepeso u obesidad a través de la medición de parámetros y la identificación de variables críticas. Por ello, se planea mostrar recomendaciones en los casos que lo requieran; por ejemplo, si la frecuencia cardíaca 
es más alta o baja de acuerdo con los valores normales, por el exceso o la falta de ejercicio, o si en la ingesta de alimentos disminuye el consumo de proteínas o se incrementa el consumo de grasas o carbohidratos.

Tratamiento. Consiste en apoyar a los usuarios con los cuidados paliativos necesarios para tratar los síntomas y los efectos secundarios causados por el sobrepeso u obesidad, además se pretende dar seguimiento a las recomendaciones realizadas para monitorizar la evolución e identificar qué recomendación es la que proporciona mejores resultados.

Para el diseño de los módulos, se presenta un diagrama UML (Unified Modeling Language) de clases, el cual muestra de forma detallada la estructura que contendrá el sistema, el cual cuenta con un motor de descargas que se encargará de obtener la información correspondiente de las API (Application Programming Interface) requeridas sin afectar el rendimiento de la aplicación, además de formular las consultas necesarias para brindar información de interés para los usuarios, médicos y a los servicios de salud que lo integren. Esta estructura como se observa en la Fig. 2, está pensada en un modelo orientado a objetos con 5 capas principales:

1. Capa de Presentación: Esta capa mantiene comunicación directa con el usuario, y contiene las siguientes clases:

- Corazón: clase específica para mantener la información correspondiente a los datos del corazón, como son número de latidos por minuto, frecuencia cardíaca, entre otros.

- Sueño: mantiene los elementos necesarios para monitorizar el tiempo y profundidad del sueño.

- Dispositivo: mantiene la estructura para el control de los dispositivos del usuario.

- Pasos: contiene el control de pasos y pisos subidos.

- Líquido: mantiene estructura necesaria para la manipulación del líquido registrado, así como la cantidad.

- Usuario: ejemplifica la estructura de almacenamiento del usuario.

- Ejercicio: corresponde a la administración del ejercicio realizado.

- Ingesta: realizado para mantener y controlar la información de ingesta diaria.

2. Capa de Integración. Esta capa está integrada por el proveedor del wearable o sensor, el componente selector de consulta y el formador de respuesta. Todos ellos colaboran para recibir datos y consultas del usuario y formular las respuestas correspondientes mediante la siguiente clase:

- Motor: encargado de controlar las consultas, conectores a servicios y ejecución de consultas, procesamiento de información y organización, esta capa preprocesa los datos para su fácil entendimiento y consumo.

3. Capa de Servicios en el IoT: Esta capa es responsable de vincular, invocar, seleccionar y confirmar los servicios en IoT, contiene la siguiente clase:

- Servicio: encargada de vincular y ejecutar los servicios médicos correspondientes.

4. Capa Analítica de Datos. Esta capa incluye el servicio de identificación de variables críticas y el sistema de recomendación médica. 
5. Capa de Datos: Contiene todos los datos útiles para la Plataforma Inteligente de Salud y está integrado por datos o eventos en tiempo real, variables críticas y recomendaciones médicas.

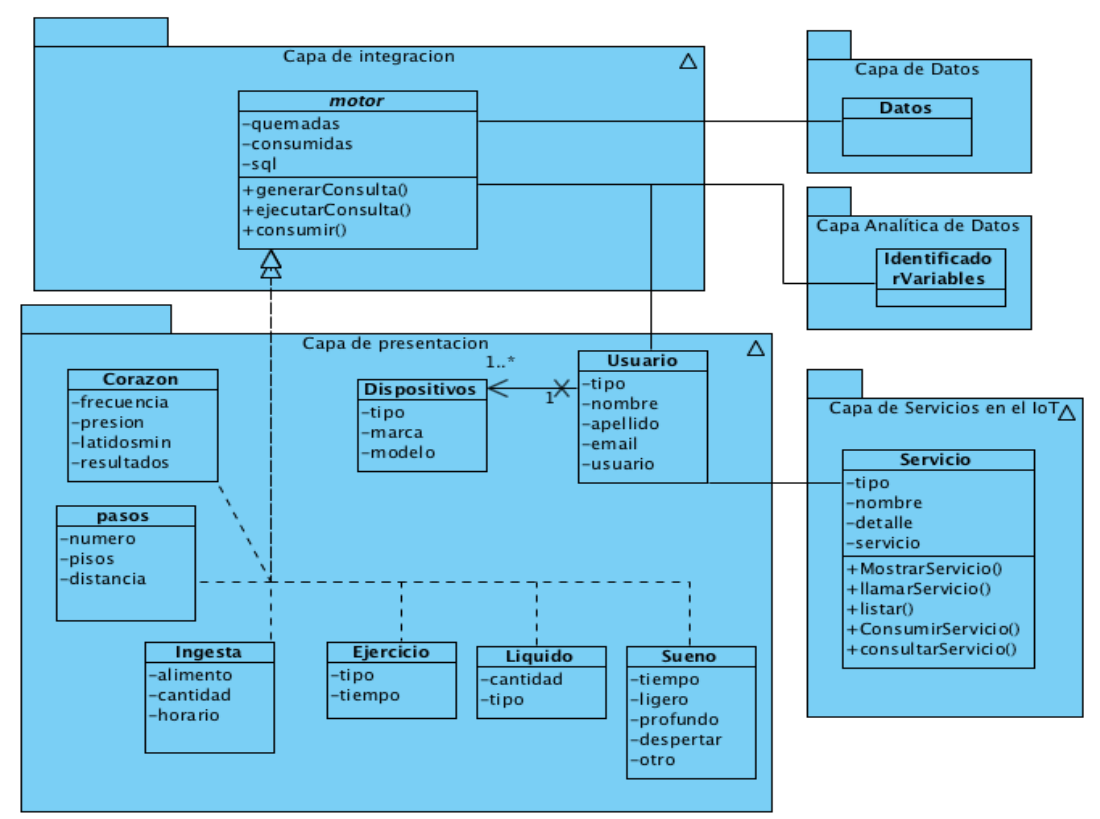

Fig. 2. Diagrama UML de clases del diseño de la arquitectura.

En la Fig. 3 se presenta un diagrama UML de paquetes, que representa la interacción de los paquetes que contienen los algoritmos de IA relacionados con el motor de procesamiento de la aplicación Web para el seguimiento y control del sobrepeso u obesidad.

Asimismo, en el diagrama UML de paquetes se presentan dos elementos que interactúan entre sí: el motor de procesamiento y los servicios en el IoT. Por otra parte, el paquete motor de procesamiento tiene conectividad con el conector de base de datos para obtener la información almacenada y con el procesador de información que se encarga de procesar los datos obtenidos por el conector y prepararlos de forma adecuada en el formato correcto, para enviarlos a los algoritmos de clasificación y recomendación.

Adicionalmente, el paquete Servicios en el IoT contiene el API de inteligencia artificial que almacena los algoritmos de clasificación y recomendación a utilizar en la aplicación Web. Los algoritmos de clasificación que se consideran utilizar son los algoritmos Naive Bayes (utilizados en la exploración inicial de los datos, buscan correlaciones entre atributos y son de entrenamiento rápido), el algoritmo C4.5 (procesa la información de una base de datos y construye diagramas lógicos) y aprendizaje profundo (emula el enfoque de aprendizaje que los seres humanos utilizan para obtener ciertos tipos de conocimiento, se considera como una forma de automatizar el análisis predictivo). 


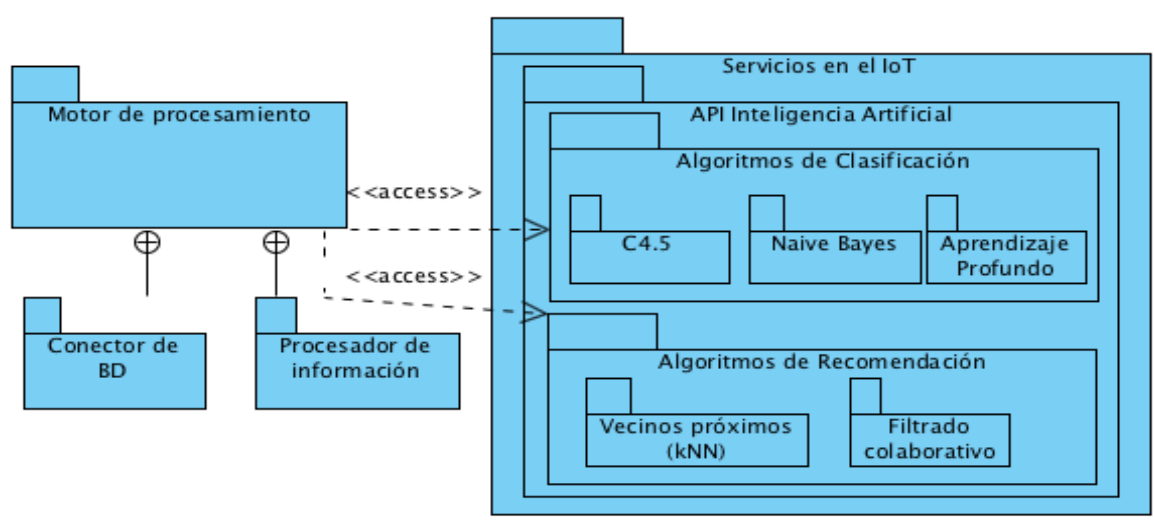

Fig. 3. Diagrama UML de paquetes que describe la interacción de los algoritmos de IA con la aplicación Web.

Tabla 2. Principales funciones de la aplicación Web relacionada con el sobrepeso u obesidad.

\begin{tabular}{|c|c|}
\hline Funcionalidad & Descripción \\
\hline ¿Cuántos pasos dados por día? & $\begin{array}{l}\text { Se obtiene la distancia recorrida y con base en eso las } \\
\text { calorías quemadas. }\end{array}$ \\
\hline ¿Cuántos pisos subidos por día? & $\begin{array}{l}\text { Por medio de esta información se obtienen las calorías } \\
\text { quemadas. }\end{array}$ \\
\hline $\begin{array}{l}\text { ¿En promedio cuantas horas se } \\
\text { duerme por día en una semana? }\end{array}$ & $\begin{array}{l}\text { Se logra obtener el promedio de sueño y relacionarlo } \\
\text { con el ritmo cardíaco y otras variables. }\end{array}$ \\
\hline $\begin{array}{l}\text { ¿Cuántos litros de agua natural se } \\
\text { consume por día? }\end{array}$ & $\begin{array}{l}\text { Con base en esta información se logra saber los niveles } \\
\text { de hidratación del cuerpo. }\end{array}$ \\
\hline $\begin{array}{l}\text { ¿En promedio cuál es el ritmo } \\
\text { cardíaco por día? }\end{array}$ & $\begin{array}{l}\text { Logra mantener monitoreado el corazón y saber si } \\
\text { existe un incremento excesivo o disminución. }\end{array}$ \\
\hline $\begin{array}{l}\text { ¿Cuántas calorías se ingieren por } \\
\text { día? }\end{array}$ & $\begin{array}{l}\text { Es necesario ya que es la base para mantener un control } \\
\text { de sobrepeso u obesidad. }\end{array}$ \\
\hline $\begin{array}{l}\text { ¿Cuántas calorías se consumieron } \\
\text { hasta el día de hoy? }\end{array}$ & $\begin{array}{l}\text { Esta información es necesaria ya que se logra obtener } \\
\text { un registro total de cuántas calorías se consumieron y } \\
\text { así poder dar una mejor recomendación. }\end{array}$ \\
\hline $\begin{array}{l}\text { ¿Cuántas calorías se consumen al } \\
\text { mes? }\end{array}$ & Necesario para mantener un control de sobrepeso. \\
\hline $\begin{array}{l}\text { ¿En promedio cuántas calorías se } \\
\text { consumen por día en una semana? }\end{array}$ & $\begin{array}{l}\text { Es necesario ya que es la base para lograr una } \\
\text { recomendación con base en calorías consumidas. }\end{array}$ \\
\hline $\begin{array}{l}\text { ¿Cuál es el tipo de ejercicio } \\
\text { realizado con mayor frecuencia? }\end{array}$ & $\begin{array}{l}\text { Dependiendo del ejercicio se logra saber cuántas } \\
\text { calorías se queman y da retroalimentación sobre gustos } \\
\text { o rutinas. }\end{array}$ \\
\hline $\begin{array}{l}\text { ¿En promedio cuántas calorías se } \\
\text { queman por cada día de una } \\
\text { semana? }\end{array}$ & $\begin{array}{l}\text { Indica de forma detallada cuántas calorías son } \\
\text { quemadas durante cada día de la semana sin tomar en } \\
\text { cuenta la actividad a la que se relaciona. }\end{array}$ \\
\hline $\begin{array}{l}\text { ¿Cuántas calorías se ingieren por } \\
\text { mes? }\end{array}$ & $\begin{array}{l}\text { Gracias a esta información se sabe cuáles son los meses } \\
\text { en los que se consumen más calorías. }\end{array}$ \\
\hline
\end{tabular}


Uriel Ramos-Deonati, Giner Alor-Hernández, Isaac Machorro-Cano, et al.

\begin{tabular}{|l|l|}
\hline \multicolumn{1}{|c|}{ Funcionalidad } & \multicolumn{1}{c|}{ Descripción } \\
\hline \hline $\begin{array}{l}\text { ¿Cuántos días de la semana se } \\
\text { encuentra por debajo de las calorías } \\
\text { consumidas en relación con las } \\
\text { calorías quemadas? }\end{array}$ & $\begin{array}{l}\text { Es importante obtener esta información ya que al } \\
\text { contrario de las anteriores se sabe si se están quemando } \\
\text { más calorías de las necesarias. }\end{array}$ \\
\hline $\begin{array}{l}\text { ¿Qué día de la semana se } \\
\text { consumieron menos calorías? }\end{array}$ & $\begin{array}{l}\text { Esta información es importante ya que da una idea de } \\
\text { qué día y en relación a que se consumieron menos } \\
\text { calorías. }\end{array}$ \\
\hline \hline $\begin{array}{l}\text { ¿Qué ejercicio produce quemar } \\
\text { más calorías? }\end{array}$ & $\begin{array}{l}\text { Dependiendo el ejercicio que se realice da un indicador } \\
\text { de qué ejercicio de los que se realizan beneficia más. }\end{array}$ \\
\hline
\end{tabular}

Por otra parte, los algoritmos de recomendación que se consideran utilizar son: el algoritmo de vecinos próximos KNN (utiliza los datos recolectados para calcular la similitud entre los elementos comunes, es sencillo, eficaz y funciona realizando la búsqueda de elementos con patrones de evaluación similares), y los algoritmos de filtrado colaborativo (mejoran el rendimiento en relación a la predicción utilizando las evaluaciones de los elementos afines para indicar una recomendación). Estos algoritmos se pretenden utilizar para la identificación de variables criticas (calorías quemadas, sueño, ritmo cardíaco, IMC, número de pisos subidos, calidad del sueño, calorías consumidas, ejercicios, control de peso) y la generación de recomendaciones.

Además, en la Tabla 2 se presentan las 15 principales funciones de la aplicación Web propuestas, relacionadas con el sobrepeso u obesidad. Los datos se presentan en relación con la estructura de la pregunta de interés a resolver que cumple el sistema y una descripción de la funcionalidad que debe realizar.

En la siguiente sección se presentan las funcionalidades de la aplicación Web para el seguimiento y control del sobrepeso u obesidad bajo el enfoque del IoT.

\section{Prototipo de la aplicación Web}

La aplicación Web propuesta proporciona un ambiente gráfico amigable que tiene el objetivo de contribuir al control del sobrepeso u obesidad y la motivación del usuario, además de mostrar datos estadísticos de la información obtenida, este diseño flexible le proporciona herramientas de registro de ingesta; asimismo, está basado en componentes para la Web y dispositivos móviles. A continuación, se presentan algunos elementos que forman parte de la propuesta.

\subsection{Visualización de la monitorización del sobrepeso u obesidad}

En la Fig. 4 se presenta la vista realizada para el apartado de monitorización, el cual se encarga de presentar datos estadísticos y diarios de un usuario, mediante 5 gráficas que presentan la información en tiempo real.

Esta vista muestra, a través de mosaicos, los datos diarios relacionados con el peso, la frecuencia de actividad física, las calorías quemadas, las calorías por consumir y el IMC, representados en gráficas que muestren la información en tiempo real. Además, 
se presentan cuatro entradas, las cuales sirven como filtros combinables, que brindan la posibilidad de obtener detalles estadísticos, por semana, por mes o incluso por año.

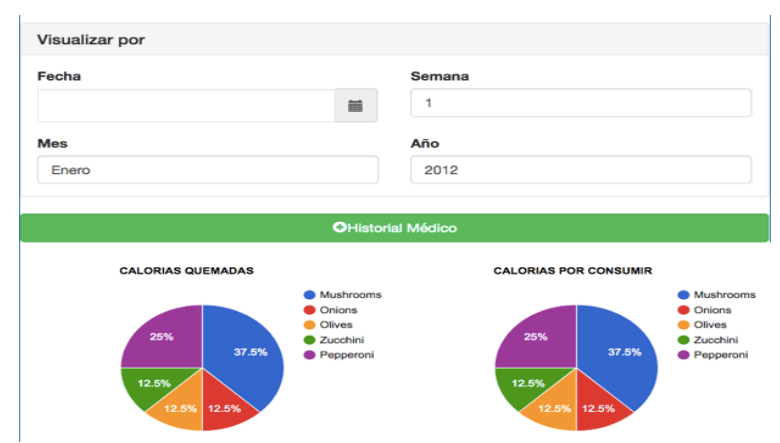

Fig. 4. Diseño de monitorización del sobrepeso y obesidad.

Además, al ingresar a la información de cada variable (peso, la frecuencia de actividad física, las calorías quemadas, las calorías por consumir y el IMC) se plantea visualizar la monitorización de los datos de cada variable para comparar el progreso por medio de una vista retrospectiva, con el propósito de identificar las variables en las que se va mejorando, en las que se mantienen los datos o en las que es necesario mejorar, para motivar e incentivar a obtener el indicador de IMC en la clasificación de normopeso.

\subsection{Sincronización de wearables}

En el diseño de la interfaz del menú "Sincronizar dispositivo" de la pestaña "Mi perfil" se utiliza un botón desplegable para seleccionar el tipo de dispositivo (Smartband, Smartwatch, Sensor, Otro); un botón desplegable para seleccionar la marca del dispositivo (Fitbit, Garmin, Polar, Samsung, Motorola, Apple, entre otros), y un botón desplegable para que, de acuerdo a la marca del dispositivo, se seleccione el modelo que le corresponda. En la Fig. 5 se presenta el diseño del apartado de la sincronización de wearables.

Adicionalmente, el apartado de sincronización de wearables muestra tres listas, las cuales se cargan en función de la opción seleccionada; primero, se debe seleccionar el tipo de dispositivo a sincronizar, seguido de la marca, la cual despliega una lista de marcas soportadas, y con base en la marca seleccionada se recarga la lista de modelos, actualizando la lista de modelos disponibles para esa marca.

Asimismo, en la parte inferior se contempla el botón "Guardar y sincronizar", para respaldar los datos seleccionados y conjuntamente sincronizar la cuenta del usuario con el o los dispositivos que realizarán el monitoreo.

\subsection{Recomendaciones}

La solución propuesta para el apartado de recomendaciones se presenta en la Fig. 6 y cuenta con la sección: Sobrepeso u obesidad. En esta subsección se muestran las 
recomendaciones de los signos vitales relacionados con el sobrepeso u obesidad (peso, calorías consumidas, calorías quemadas, actividad física, IMC).

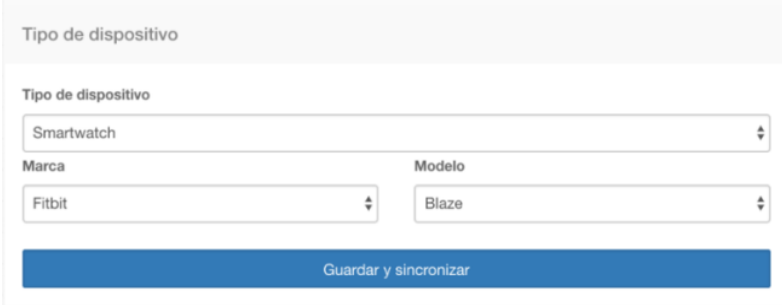

Fig. 5. Diseño del apartado de Sincronización de wearables.

También, en este apartado se presenta una serie de recomendaciones para el usuario, obtenidas con base en los datos almacenados utilizando algoritmos de inteligencia artificial, tales como el algoritmo de clasificación C4.5, el cual es un modelo de predicción y una extensión del algoritmo ID3, que soluciona algunos de sus inconvenientes. Asimismo, el algoritmo C4.5 procesa la información de una base de datos y construye diagramas lógicos, similares a los que se obtienen en sistemas de predicción basados en reglas, que sirven para representar y categorizar una serie de condiciones que suceden de forma sucesiva, para la resolución de un problema. Este tipo de modelos se utilizan para predecir el valor de un atributo categórico (discreto o nominal) y su aplicación se realiza en diagnósticos médicos.

Adicionalmente, se plantea utilizar alternativas de software de aprendizaje automático y minería de datos que ofrecen algunas API de integración (Weka [16] y Apache Mahout [17]), las cuales se planea integrarlas al sistema con la finalidad de realizar recomendaciones por medio del análisis de la información almacenada.

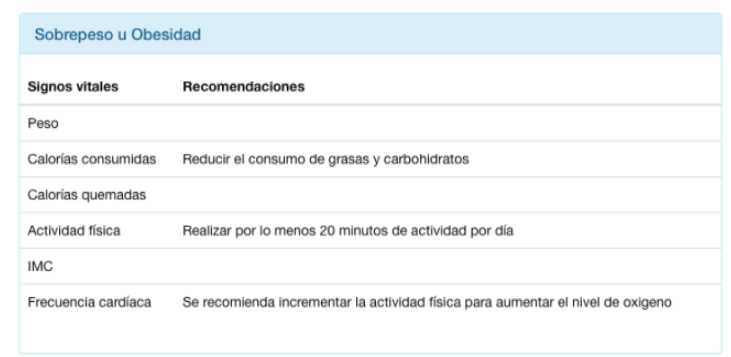

Fig. 6. Vista del apartado recomendaciones

Además, al realizar un análisis comparativo de las API se identificó que uno de los aspectos relevantes en las plataformas es el tipo de licencia, ya que todas tienen un API de desarrollo, algunos ofrecen versiones comunitarias incompletas, como son Rapid miner [18] y KNIME [19], pero en otros casos existen versiones libres como Weka [16] y Apache Mahout [17].

Por otro lado, el procesamiento de información se pretende realizar utilizando los datos históricos obtenidos por los wearables. Una vez almacenada la información en la base de datos la aplicación Web envía los datos por medio de un servicio 
RESTFUL para el análisis y obtención de recomendaciones. Además, se plantea que las recomendaciones realizadas estén validadas por especialistas en el área de la salud.

En la siguiente sección se presentan las conclusiones a las que se llegaron al realizar el diseño de la aplicación Web para el seguimiento y control del sobrepeso u obesidad bajo el paradigma del IoT. Además, se plantea el trabajo a futuro y los agradecimientos.

\section{Conclusiones y trabajo a futuro}

El sobrepeso y la obesidad son problemas de salud transversales que afectan la calidad de vida de las personas, incluida su vida laboral. En este sentido, el IoT brinda grandes oportunidades para monitorear, analizar, diagnosticar, controlar y brindar recomendaciones de tratamiento para enfermedades crónico-degenerativas, como el sobrepeso u obesidad.

En este trabajo se propone el diseño de una aplicación Web que permita el seguimiento y, posteriormente, el control del sobrepeso u obesidad bajo el enfoque del IoT, incorporando técnicas de inteligencia artificial, aplicadas al cuidado de la salud. De manera similar, se discutió cómo, a través de varios trabajos de investigación, el IoT contribuye al diagnóstico y tratamiento de enfermedades como el sobrepeso u obesidad.

Como trabajo futuro se realizará un análisis comparativo de los trabajos más relevantes que abordan el papel del IoT en la prevención, el control, el tratamiento y la lucha contra el sobrepeso u obesidad. Además, se busca validar la aplicación Web para el seguimiento y control del sobrepeso u obesidad bajo el paradigma del IoT, en un caso de estudio de atención médica y, posteriormente, desarrollar la aplicación Web para el seguimiento y tratamiento del sobrepeso u obesidad de un paciente basado en el IoT.

Agradecimientos. Los autores agradecen al Consejo Nacional de Ciencia y Tecnología (CONACYT), al Tecnológico Nacional de México (TecNM), al Programa para el Desarrollo Profesional Docente (PRODEP) y a la Universidad del Papaloapan (UNPA) por el apoyo otorgado para la realización de esta investigación.

\section{Referencias}

1. Obesity and overweight, http://www.who.int/mediacentre/factsheets/fs311/en/ (2018)

2. Ling, Li, Shancang, Li, Shanshan, Z.: QoS-Aware Scheduling of Services-Oriented Internet of Things. IEEE Transactions on Industrial Informatics 10(2), pp. 1497-1505 (2014)

3. Xu, L., He, W., Li, S.: Internet of Things in Industries: A Survey. IEEE Transactions on Industrial Informatics 10(4), pp. 2233-2243 (2014)

4. Vilallonga, R., Lecube, A., Fort, J., Boleko, M., Hidalgo, M., Armengol, M.: Internet of Things and bariatric surgery follow-up: Comparative study of standard and IoT follow-up. Minimally Invasive Therapy \& Allied Technologies 22(5), pp. 304-311 (2013) 
5. Zaragozá, I., Guixeres, J., Alcañiz, M., Cebolla, A., Saiz, J., Álvarez, J.: Ubiquitous monitoring and assessment of childhood obesity. Personal and Ubiquitous Computing 17(6), pp. 1147-1157 (2012)

6. Vazquez, M., Jimenez, E., Nieto, J., Sanchez, J., Garcia, A., Torres, J.: Development of a Mobile Health Architecture to Prevent Childhood Obesity. IEEE Latin America Transactions 13(5), pp. 1520-1527 (2015)

7. Kim, K., Logan, H., Young, E., Sabee, C.: Youth-centered design and usage results of the iN Touch mobile self-management program for overweight/obesity. Personal and Ubiquitous Computing 19(1), pp. 59-68 (2014)

8. Alloghani, M., Hussain, A., Al-Jumeily, D., Fergus, P., Abuelma'atti, O., Hamden, H.: A mobile health monitoring application for obesity management and control using the internet-of-things. In: Sixth International Conference on Digital Information Processing and Communications (ICDIPC), pp. 19-24 (2016)

9. Wibisono, G., Astawa, I.: Designing Machine-to-Machine (M2M) Prototype System for Weight Loss Program for Obesity and Overweight Patients. In: International Conference on Intelligent Systems, Modelling and Simulation (ISMS), pp. 1197-209 (2016)

10. Aupetit, M., Fernandez-Luque, L., Singh, M., Srivastava, J.: Visualization of Wearable Data and Biometrics for Analysis and Recommendations in Childhood Obesity. In: International Symposium on Computer-Based Medical Systems (CBMS), pp. 678-679 (2017)

11. Yang, H., Kang, J., Kim, O., Choi, M., Oh, M., Nam, J., Sung, E.: Interventions for Preventing Childhood Obesity with Smartphones and Wearable Device: A Protocol for a Non-Randomized Controlled Trial. International Journal of Environmental Research and Public Health 14(2), p. 184 (2017)

12. Laing, B., Mangione, C., Tseng, C., Leng, M., Vaisberg, E., Mahida, M., Bholat, M., Glazier, E., Morisky, D., Bell, D.: Effectiveness of a Smartphone Application for Weight Loss Compared with Usual Care in Overweight Primary Care Patients. Annals of Internal Medicine 161, pp. S5-S12 (2014)

13. Otto, C., Milenković, A., Sanders, C., Jovanov, E.: System Architecture of a Wireless Body Area Sensor Network for Ubiquitous Health Monitoring: Journal of Mobile Multimedia, vol. 1, pp 307-326 (2006)

14. Ahmed, M., Björkman, M., Čaušević, A., Fotouhi, H., Lindén, M.: An Overview on the Internet of Things for Health Monitoring Systems. Internet of Things. IoT Infrastructures. pp. 429-436 (2016)

15. Shin, S.A., Lee, N.Y., Park, J.H.: Empirical study of the IoT-learning for obese patients that require personal training: Advances in Computer Science and Ubiquitous Computing. LNEE, vol. 421, pp. 1005-1012. Springer, Singapore (2017)

16. Weka 3 - Data Mining with Open Source Machine Learning Software in Java (2018)

17. Apache Mahout (2018)

18. Data Science Platform | RapidMiner (2018)

19. KNIME - Open for Innovation (2018) 